\title{
A CLASS OF SASAKIAN 5-MANIFOLDS
}

\author{
ADRIÁN ANDRADA, ANNA FINO AND LUIGI VEZZONI
}

\begin{abstract}
We obtain some general results on Sasakian Lie algebras and prove as a consequence that a $(2 n+1)$-dimensional nilpotent Lie group admitting left-invariant Sasakian structures is isomorphic to the real Heisenberg group $H_{2 n+1}$. Furthermore, we classify Sasakian Lie algebras of dimension 5 and determine which of them carry a Sasakian $\alpha$-Einstein structure. We show that a 5 -dimensional solvable Lie group with a left-invariant Sasakian structure and which admits a compact quotient by a discrete subgroup is isomorphic to either $H_{5}$ or a semidirect product $\mathbb{R} \ltimes\left(H_{3} \times \mathbb{R}\right)$. In particular, the compact quotient is an $S^{1}$-bundle over a 4 -dimensional Kähler solvmanifold.
\end{abstract}

\section{INTRODUCTION}

A Sasakian structure is the analogous in odd dimensions of a Kähler structure. Indeed, by [5] a Riemannian manifold $(M, g)$ of odd dimension $2 n+1$ admits a compatible Sasakian structure if and only if the Riemannian cone $M \times \mathbb{R}^{+}$is Kähler.

In dimension 3 a homogeneous Sasakian manifold has to be a Lie group endowed with a left-invariant Sasakian structure by [27]. Therefore the classification of 3-dimensional Sasakian homogeneous spaces depends on the classification of 3-dimensional Sasakian Lie algebras.

By [26] a compact, simply connected, 5-dimensional homogeneous contact manifold is diffeomorphic to the 5-dimensional sphere $S^{5}$ or to the product of two spheres $S^{2} \times S^{3}$. Moreover, both $S^{5}$ and $S^{2} \times S^{3}$ carry Sasakian-Einstein structures (see [7, 24]). Other explicit examples of Sasakian-Einstein 5-manifolds have been found in [16], while toric Sasakian manifolds in dimension 5 have been studied in 9] and [11. A classification of Sasakian-Einstein 5-manifolds of cohomogeneity 1 has been obtained in [12 and a classification of 5 -dimensional Lie groups endowed with a left-invariant contact structure was obtained in [14.

As far as we know in the literature the only result about 5-dimensional Lie groups admitting left-invariant Sasakian structures is in the case of nilpotent Lie groups. Indeed, in [32] it was shown that the only 5-dimensional nilpotent Sasakian Lie algebra is the real Heisenberg Lie algebra $\mathfrak{h}_{5}$. It was proved in [9] that the real Heisenberg Lie group $H_{2 n+1}$ admits no bi-invariant Sasakian structure, even if it has a left-invariant Sasakian structure as well as a right-invariant one. These two structures are different.

The aim of this paper is to classify 5-dimensional Lie groups endowed with a leftinvariant Sasakian structure. This is equivalent to determining all 5-dimensional Sasakian

Date: November 1, 2018.

1991 Mathematics Subject Classification. 53C25, 22E60, 53C30.

This work was partially supported by Conicet, ANPCyT, Secyt-UNC (Argentina), MIUR, GNSAGA (Italy) and by the WWS Project "Geometria Riemanniana e Gruppi di Lie". 
Lie algebras. The study of Sasakian Lie algebras is done taking into account the center of the Lie algebra, which can only be trivial or 1-dimensional. In this way we obtain some general results on Sasakian Lie algebras in any dimensions. In particular, we show that the only $(2 n+1)$-dimensional nilpotent Lie algebra admitting a Sasakian structure is the real Heisenberg Lie algebra $\mathfrak{h}_{2 n+1}$.

By using the previous general results and independently from the list obtained in [14], we obtain a classification up to isomorphism of 5-dimensional Sasakian Lie algebras.

Main Theorem. Let $\mathfrak{g}$ be a 5-dimensional Lie algebra admitting a Sasakian structure. Then

- if $\mathfrak{g}$ has non-trivial center $\mathfrak{z}(\mathfrak{g})$, then $\mathfrak{g}$ is solvable with $\operatorname{dim} \mathfrak{z}(\mathfrak{g})=1$ and the quotient $\mathfrak{g} / \mathfrak{z}(\mathfrak{g})$ carries an induced Kähler structure (see Theorem 4.1);

- if $\mathfrak{g}$ has trivial center, then it is isomorphic to one of the following Lie algebras: the direct products $\mathfrak{s l}(2, \mathbb{R}) \times \mathfrak{a f f}(\mathbb{R}), \mathfrak{s u}(2) \times \mathfrak{a f f}(\mathbb{R})$, or the non-unimodular solvable Lie algebra $\mathfrak{g}_{0} \cong \mathbb{R}^{2} \ltimes \mathfrak{h}_{3}$ with structure equations

$$
\begin{array}{lll}
{\left[e_{1}, e_{3}\right]=e_{3},} & {\left[e_{1}, e_{4}\right]=\frac{1}{2} e_{4},} & {\left[e_{1}, e_{5}\right]=\frac{1}{2} e_{5},} \\
{\left[e_{2}, e_{4}\right]=e_{5},} & {\left[e_{2}, e_{5}\right]=-e_{4}, \quad\left[e_{4}, e_{5}\right]=-e_{3},}
\end{array}
$$

where $\mathfrak{a f f}(\mathbb{R})$ is the Lie algebra of the Lie group of affine motions of $\mathbb{R}$ and $\mathfrak{h}_{3}$ is the real 3-dimensional Heisenberg Lie algebra (see Theorem 4.4).

As a consequence we obtain that $\mathfrak{g}$ is either solvable or a direct product of a 3dimensional semisimple ideal with the radical $\mathfrak{a f f}(\mathbb{R})$.

In the case of non-trivial center we determine the list of the 5-dimensional Sasakian Lie algebras by using the classification of 4-dimensional Kähler Lie algebras given by Ovando in $[25$.

Moreover, we prove that the only 5-dimensional simply connected Lie groups with a left-invariant Sasakian structure which admit a compact quotient by a discrete subgroup are the real Heisenberg group $H_{5}$ or a semidirect product $\mathbb{R} \ltimes\left(\mathbb{R} \times H_{3}\right)$ (see Corollary 4.2). By [21] a solvmanifold, i.e. a compact quotient of a solvable Lie group by a discrete subgroup, endowed with a Kähler structure is a finite quotient of a complex torus. We show that a compact quotient of a 5 -dimensional solvable Lie group with a left-invariant Sasakian structure by a uniform discrete subgroup is an $S^{1}$-bundle over a 4 -dimensional Kähler solvmanifold.

By [1, Proposition 4.2] $\mathfrak{g}_{0}$ is the only solvable (non-nilpotent) 5-dimensional Lie algebra admitting a Sasakian $\alpha$-Einstein structure. We show that a 5-dimensional Sasakian $\alpha$ Einstein Lie algebra is isomorphic either to $\mathfrak{h}_{5}, \mathfrak{g}_{0}$ or to $\mathfrak{s l}(2, \mathbb{R}) \times \mathfrak{a f f}(\mathbb{R})$.

Moreover, by [15] it is known that a Lie algebra of dimension at least 5 cannot admit a Sasakian-Einstein structure.

Acknowledgements We would like to thank Liviu Ornea for useful comments and the referees for helpful suggestions and remarks.

\section{Preliminaries}

A triple $(\Phi, \alpha, \xi)$ on a $(2 n+1)$-dimensional manifold $M$ is an almost contact structure if $\xi$ is a nowhere vanishing vector field, $\alpha$ is a 1 -form, and $\Phi$ is a tensor of type $(1,1)$ such 
that

$$
\alpha(\xi)=1, \quad \Phi^{2}=-\mathrm{I}+\xi \otimes \alpha .
$$

The vector field $\xi$ defines the characteristic foliation $\mathcal{F}$ with 1-dimensional leaves, and the kernel of $\alpha$ defines the codimension one sub-bundle $\mathcal{D}=\operatorname{ker} \alpha$. Then there is the canonical splitting of the tangent bundle $T M$ of $M$

$$
T M=\mathcal{D} \oplus \mathcal{L},
$$

where $\mathcal{L}$ is the trivial line bundle generated by $\xi$. Note that conditions (2) imply

$$
\Phi(\xi)=0, \quad \alpha \circ \Phi=0 .
$$

If the 1 -form $\alpha$ satisfies the condition

$$
\alpha \wedge(\mathrm{d} \alpha)^{n} \neq 0
$$

then the subbundle $\mathcal{D}$ defines a contact structure on $M$. In this case the vector field $\xi$ is called the Reeb vector field and $\alpha$ is called a contact form. Contact structures can be considered as the odd-dimensional counterpart of symplectic structures. If $\alpha$ is a contact form, then the associated Reeb vector field satisfies

$$
\mathrm{d} \alpha(\xi, X)=0
$$

for any vector field $X$ on $M$.

Similarly to the case of an almost complex structure, there is the notion of integrability of an almost contact structure. Indeed, an almost contact structure $(\Phi, \alpha, \xi)$ is called normal if the Nijenhuis tensor $N_{\Phi}$ associated to the tensor $\Phi$ defined by

$$
N_{\Phi}(X, Y)=\Phi^{2}[X, Y]+[\Phi X, \Phi Y]-\Phi[\Phi X, Y]-\Phi[X, \Phi Y],
$$

satisfies the condition

$$
N_{\Phi}=-\mathrm{d} \alpha \otimes \xi .
$$

This last condition is equivalent to requiring that the almost complex structure

$$
J\left(X, f \frac{\partial}{\partial t}\right)=\left(\Phi X-f \xi, \alpha(X) \frac{\partial}{\partial t}\right)
$$

on the product $M \times \mathbb{R}$ be integrable, where $f$ is a smooth function on $M \times \mathbb{R}$ and $t$ is the coordinate on $\mathbb{R}$ (see [30]).

A Riemannian metric $g$ on an almost contact manifold $(M, \Phi, \alpha, \xi)$ is compatible with the almost contact structure if

$$
g(\Phi X, \Phi Y)=g(X, Y)-\alpha(X) \alpha(Y),
$$

for any vector fields $X, Y$. In this case the structure $(\Phi, \alpha, \xi, g)$ is called an almost contact metric structure. Any almost contact structure admits a compatible metric.

An almost contact metric structure $(\Phi, \alpha, \xi, g)$ is said to be contact metric if

$$
2 g(X, \Phi Y)=\mathrm{d} \alpha(X, Y) .
$$

In this case $\alpha$ is a contact form and we denote

$$
\omega(X, Y)=g(X, \Phi Y) .
$$


Definition 2.1. 28, A Sasakian structure is a normal contact metric structure, i.e. an almost contact metric structure $(\Phi, \alpha, \xi, g)$ such that

$$
N_{\Phi}=-\mathrm{d} \alpha \otimes \xi, \quad \mathrm{d} \alpha=2 \omega .
$$

A Sasakian structure can be also characterized in terms of the Riemannian cone over the manifold. More precisely, we recall that a Riemannian manifold $(M, g)$ admits a compatible Sasakian structure if and only if the cone $M \times \mathbb{R}^{+}$equipped with the metric $h=t^{2} g+\mathrm{d} t \otimes \mathrm{d} t$ is Kähler (see for instance [5]). Furthermore, in this case the Reeb vector field is Killing and the covariant derivative of $\Phi$ with respect to the Levi-Civita connection of $g$ is given by

$$
\left(\nabla_{X} \Phi\right)(Y)=g(\xi, Y) X-g(X, Y) \xi
$$

for any pair of vector fields $X$ and $Y$ on $M$ (see for instance [5]).

If a $(2 n+1)$-dimensional manifold $M$ admits a Sasakian structure, the product metric on $M \times \mathbb{R}$ is compatible with the complex structure $J$ given by (6) and moreover by [33, Proposition 3.5] the corresponding Hermitian structure is locally conformally Kähler with parallel Lee form.

A contact metric structure $(\Phi, \alpha, \xi, g)$ is Sasakian if and only if its Riemannian curvature tensor satisfies the condition

$$
R(X, Y) \xi=\alpha(Y) X-\alpha(X) Y
$$

for any vector fields $X$ and $Y$ (see for instance [2]). This implies that the Ricci tensor $\mathrm{Ric}_{g}$ associated to a Sasakian metric satisfies

$$
\operatorname{Ric}_{g}(\xi, X)=2 n \alpha(X)
$$

for any vector field $X$ on $M$, where $\operatorname{dim} M=2 n+1$. In particular $\operatorname{Ric}_{g}(\xi, \xi)=2 n$ and the metric $g$ is never Ricci-flat.

When the Ricci curvature tensor is given by $\operatorname{Ric}_{g}=\lambda g+\nu \alpha \otimes \alpha$, for some constants $\lambda, \nu \in \mathbb{R}$, the Sasakian structure is called $\alpha$-Einstein $([23,29,8])$. In his original definition Okumura assumed that both $\lambda$ and $\nu$ are functions, and then he showed, as for the Einstein metrics, that they must be constant when the dimension of the manifold is greater than three. When $\nu=0$, the Sasakian structure is called Sasakian-Einstein. These structures have been intensively studied by many authors (see for instance [6, 7, 16, 17] and references therein). Finally, we recall that a 5-dimensional manifold is Sasakian $\alpha$-Einstein if and only if it is Sasakian-hypo (see [13]).

\section{SASAKian Lie Algebras}

In this section we will begin our study of left-invariant Sasakian structures on Lie groups. Such a structure corresponds to a Sasakian structure on the associated Lie algebra.

Definition 3.1. A Sasakian structure on a Lie algebra $\mathfrak{g}$ is a quadruple $(\Phi, \alpha, \xi, g)$, where $\Phi \in \operatorname{End}(\mathfrak{g}), \alpha \in \mathfrak{g}^{*}, \xi \in \mathfrak{g}$ and $g$ is an inner product on $\mathfrak{g}$ such that

$$
\begin{aligned}
& \alpha(\xi)=1, \quad \Phi^{2}=-\mathrm{I}+\xi \otimes \alpha, \quad g(\Phi X, \Phi Y)=g(X, Y)-\alpha(X) \alpha(Y), \\
& 2 g(X, \Phi Y)=\mathrm{d} \alpha(X, Y), \quad N_{\Phi}=-\mathrm{d} \alpha \otimes \xi,
\end{aligned}
$$


where $N_{\Phi}$ is defined as in (5). A Lie algebra equipped with a Sasakian structure will be called a Sasakian Lie algebra. The vector $\xi$ will be called the Reeb vector.

Remark 3.2. We note that, in this setting, formula (41) reads

$$
\alpha(\xi, X)=0
$$

for any $X \in \mathfrak{g}$.

Example 3.3. The classical example of a Sasakian Lie algebra is given by the $(2 n+1)$-dimensional real Heisenberg Lie algebra $\mathfrak{h}_{2 n+1}$. We recall that $\mathfrak{h}_{2 n+1}=$ $\operatorname{Span}\left\{X_{1}, \ldots, X_{n}, Y_{1}, \ldots, Y_{n}, Z\right\}$, where

$$
\left[X_{i}, Y_{i}\right]=Z,
$$

for $i=1, \ldots, n$; in this case, a Sasakian structure is defined by

$$
\Phi\left(X_{i}\right)=Y_{i}, \Phi\left(Y_{i}\right)=-X_{i}, \Phi(Z)=0, i=1, \ldots, n,
$$

the inner product $g$ is obtained by declaring the basis above orthonormal, $\xi=Z$ and $\alpha$ is the dual 1-form of $Z$ with respect to the metric $g$.

In general for a Lie algebra $\mathfrak{g}$ with a contact structure $\alpha$ we can prove the following property for its center $\mathfrak{z}(\mathfrak{g})$.

Proposition 3.4. Let $(\mathfrak{g}, \alpha)$ be a contact Lie algebra with $\xi$ its Reeb vector and let $\mathfrak{z}(\mathfrak{g})$ be the center of $\mathfrak{g}$. Then

1. $\operatorname{dim} \mathfrak{z}(\mathfrak{g}) \leq 1$;

2. if $\operatorname{dim} \mathfrak{z}(\mathfrak{g})=1$, then $\mathfrak{z}(\mathfrak{g})=\mathbb{R} \xi$.

Proof. The first item is well known and follows from the fact that $\mathrm{d} \alpha$ is non-degenerate on $\operatorname{ker} \alpha$. For the second item we fix an arbitrary generator $Z$ of $\mathfrak{z}(\mathfrak{g})$. We may write $Z=a \xi+X$, where $a \in \mathbb{R}$ and $X \in \operatorname{ker} \alpha$. We have

$$
0=\alpha([Z, Y])=\alpha([a \xi+X, Y])=\alpha([X, Y])=-\mathrm{d} \alpha(X, Y)
$$

for all $Y \in \operatorname{ker} \alpha$. By the non-degeneracy of $\mathrm{d} \alpha$ on $\operatorname{ker} \alpha$, it follows that $X=0$ and, consequently, $\mathfrak{z}(\mathfrak{g})=\mathbb{R} \xi$.

Remark 3.5. We recall that due to [4, Theorem 5], the only semisimple Lie algebras admitting a contact form are $\mathfrak{s u}(2)$ and $\mathfrak{s l}(2, \mathbb{R})$.

3.1. Non-trivial center. We show that in the case of Sasakian Lie algebras with nontrivial center the kernel of the contact form inherits a natural structure of Kähler Lie algebra. Moreover two Sasakian Lie algebras are isomorphic if and only if the corresponding Kähler Lie algebras are equivalent. This allows us to use the classification of the 4-dimensional Kähler Lie algebras of [25] to classify 5-dimensional Sasakian Lie algebras with non-trivial center.

We start by considering the following

Proposition 3.6. Let $(\mathfrak{g}, \Phi, \alpha, \xi, g)$ be a Sasakian Lie algebra with non-trivial center $\mathfrak{z}(\mathfrak{g})$ generated by $\xi$. Then the quadruple $(\operatorname{ker} \alpha, \theta, \Phi, g)$ is a Kähler Lie algebra, where $\theta$ is the component of the Lie bracket of $\mathfrak{g}$ on $\operatorname{ker} \alpha$. 
Proof. Let $X, Y, Z$ in $\operatorname{ker} \alpha$, then

$$
\begin{aligned}
0= & {[X,[Y, Z]]+[Z,[X, Y]]+[Y,[Z, X]] } \\
= & {[X, \theta(Y, Z)+\alpha([Y, Z]) \xi]+[Z, \theta(X, Y)+\alpha([X, Y]) \xi] } \\
& +[Y, \theta(Z, X)+\alpha([Z, X]) \xi] \\
= & {[X, \theta(Y, Z)]+[Z, \theta(X, Y)]+[Y, \theta(Z, X)] } \\
= & \theta(X, \theta(Y, Z))+\theta(Z, \theta(X, Y))+\theta(Y, \theta(Z, X)) \\
& +\alpha([X, \theta(Y, Z)]+[Z, \theta(X, Y)]+[Y, \theta(Z, X)]) \xi
\end{aligned}
$$

i.e.

$$
\theta(X, \theta(Y, Z))+\theta(Z, \theta(X, Y))+\theta(Y, \theta(Z, X))=0, \quad \mathrm{~d} \omega=0,
$$

$2 \omega$ being the restriction of $\mathrm{d} \alpha$ on $\operatorname{ker} \alpha$. Then $\theta$ defines a Lie bracket on ker $\alpha$. Since $\omega$ is non-degenerate, the statement holds.

Conversely, let $\left(\mathfrak{h},[,]_{\mathfrak{h}}, \omega, \Phi, g\right)$ be a Kähler Lie algebra and set $\mathfrak{g}=\mathfrak{h} \oplus \mathbb{R} \xi$. Then defining

$$
[X, Y]=[X, Y]_{\mathfrak{h}}-\omega(X, Y) \xi
$$

for $X, Y \in \mathfrak{h}$ and

$$
[\xi, \mathfrak{h}]=0
$$

we obtain a new Lie algebra $(\mathfrak{g},[]$,$) equipped with a natural Sasakian structure, where$ the contact form $\alpha$ on $\mathfrak{g}$ is defined as

$$
\alpha(a \xi+X)=a
$$

for all $X \in \mathfrak{h}$ and $\Phi$ and $g$ are extended in a natural way.

Corollary 3.7. Let $(\mathfrak{g}, \Phi, \alpha, \xi, g)$ be a Sasakian Lie algebra with non-trivial center $\mathfrak{z}(\mathfrak{g})$ generated by $\xi$. Then $\mathfrak{g} / \mathfrak{z}(\mathfrak{g})$ inherits a natural Kähler structure.

Now we have the following easy-to-prove proposition which will be used in 4 .

Proposition 3.8. Two Sasakian Lie algebras with non-trivial center $\left(\mathfrak{g}_{1}, \Phi_{1}, \alpha_{1}, \xi_{1}, g_{1}\right)$, $\left(\mathfrak{g}_{2}, \Phi_{2}, \alpha_{2}, \xi_{2}, g_{2}\right)$ are isomorphic if and only ker $\alpha_{1}$ and $\operatorname{ker} \alpha_{2}$ are isomorphic as Kähler Lie algebras.

Since a nilpotent Lie algebra has always non-trivial center, we can apply the results above in order to determine all nilpotent Lie algebras admitting a Sasakian structure. It is known that in dimensions 3 and 5 the only nilpotent Sasakian Lie algebras are the real Heisenberg algebras $\mathfrak{h}_{3}$ and $\mathfrak{h}_{5}$, respectively (see [18] and [32, Corollary 5.5]). We show next that this still holds in any dimension.

Theorem 3.9. Let $\mathfrak{g}$ be a $(2 n+1)$-dimensional nilpotent Lie algebra admitting a Sasakian structure. Then $\mathfrak{g}$ is isomorphic to the $(2 n+1)$-dimensional Heisenberg Lie algebra.

Proof. Let $(\Phi, \alpha, \xi, g)$ be a Sasakian structure on $\mathfrak{g}$. Since $\mathfrak{g}$ is nilpotent it has non-trivial center $\mathfrak{z}(\mathfrak{g})=\mathbb{R} \xi$. The quotient $\mathfrak{g} / \mathfrak{z}(\mathfrak{g})$ is a Kähler nilpotent Lie algebra, hence it is unimodular, and therefore, using a result of Hano [22], it is flat. As a consequence, $\mathfrak{g} / \mathfrak{z}(\mathfrak{g})$ is abelian. This implies immediately that $\mathfrak{g}$ is isomorphic to the Heisenberg Lie algebra. 
Remark 3.10. According to 31 the $(2 n+1)$-dimensional Heisenberg Lie algebra admits a contact Calabi-Yau structure. In particular $\mathfrak{h}_{2 n+1}$ admits Sasakian $\alpha$-Einstein structures.

3.2. Trivial center. In the case the Sasakian Lie algebra $\mathfrak{g}$ has trivial center, we have the following properties for $\operatorname{ad}_{\xi}$.

Proposition 3.11. Let $(\mathfrak{g}, \Phi, \alpha, \xi, g)$ be a Sasakian Lie algebra. Then

1. $\operatorname{ad}_{\xi} \Phi=\Phi \operatorname{ad}_{\xi}$, and therefore $\operatorname{ker} \operatorname{ad}_{\xi}$ and $\operatorname{Imad}_{\xi}$ are $\Phi$-invariant subspaces of $\mathfrak{g} ;$

2. $\operatorname{ad}_{\xi} \Phi$ is symmetric with respect to $g$;

3. $\operatorname{ad}_{\xi}$ is skew-symmetric with respect to $g$ and therefore $\left(\operatorname{Im~ad}_{\xi}\right)^{\perp}=\operatorname{ker} \operatorname{ad}_{\xi}$.

Proof. The first item is an easy consequence of $N_{\Phi}=-\mathrm{d} \alpha \otimes \xi$ and (3). Indeed, for any $X \in \mathfrak{g}$,

$$
\begin{aligned}
0=N_{\Phi}(\xi, X) & =[\Phi \xi, \Phi X]-\Phi[\Phi \xi, X]-\Phi[\xi, \Phi X]+\Phi^{2}[\xi, X] \\
& =-\Phi[\xi, \Phi X]-[\xi, X]
\end{aligned}
$$

i.e.

$$
[\xi, \Phi X]=\Phi[\xi, X]
$$

The second item follows from the following computation, for $X, Y \in \mathfrak{g}$,

$$
\begin{aligned}
2 g([\xi, \Phi X], Y) & =2 g(\Phi[\xi, X], Y)=-2 g([\xi, X], \Phi Y)=-\mathrm{d} \alpha([\xi, X], Y) \\
& =\alpha([[\xi, X], Y])=-\alpha([[X, Y], \xi]-[[\xi, Y], X]) \\
& =\alpha([[\xi, Y], X])=-\mathrm{d} \alpha([\xi, Y], X)=-2 g([\xi, Y], \Phi X) \\
& =2 g(X,[\xi, \Phi Y]) .
\end{aligned}
$$

Finally, let $X, Y \in \mathfrak{g}$. We can write $X=a \xi+\Phi X^{\prime}$, with $X^{\prime} \in \operatorname{ker} \alpha$, and thus the third item follows from

$$
\begin{aligned}
g([\xi, X], Y) & =g\left(\left[\xi, a \xi+\Phi X^{\prime}\right], Y\right)=g\left(\left[\xi, \Phi X^{\prime}\right], Y\right)=g\left(X^{\prime},[\xi, \Phi Y]\right) \\
& =-g\left(\Phi X^{\prime},[\xi, Y]\right)=-g(X-a \xi,[\xi, Y])=-g(X,[\xi, Y]) .
\end{aligned}
$$

This shows also that $\operatorname{ker} \operatorname{ad}_{\xi} \subseteq\left(\operatorname{Im~ad}_{\xi}\right)^{\perp}$. For dimensional reasons we have that $\operatorname{ker} \operatorname{ad}_{\xi}=$ $\left(\operatorname{Im} \operatorname{ad}_{\xi}\right)^{\perp}$.

As a direct consequence of Proposition 3.11 we have the following

Corollary 3.12. Let $(\mathfrak{g}, \Phi, \alpha, \xi, g)$ be a Sasakian Lie algebra. Then there is an orthogonal decomposition

$$
\mathfrak{g}=\operatorname{ker} \operatorname{ad}_{\xi} \oplus \operatorname{Im} \operatorname{ad}_{\xi} .
$$

Proposition 3.13. Let $(\mathfrak{g}, \Phi, \alpha, \xi, g)$ be a Sasakian Lie algebra with trivial center.

(1) If $\operatorname{dim} \mathfrak{g} \geq 5$, then $\operatorname{ker}_{\xi}$ is a Sasakian Lie subalgebra of $\mathfrak{g}$ with non-trivial center.

(2) If $X \in \operatorname{ker} \operatorname{ad}_{\xi}, Y \in \operatorname{Imad}_{\xi}$, then $[X, Y] \in \operatorname{Imad}_{\xi}$.

Proof. (1) Since $\operatorname{ad}_{\xi}$ is a derivation, its kernel is a Lie subalgebra of $\mathfrak{g}$. Furthermore, let $X \in \operatorname{ker} \alpha \cap \operatorname{ker} \operatorname{ad}_{\xi}$ and $Y \in \mathfrak{g}$, then

$$
\mathrm{d} \alpha\left(X, \operatorname{ad}_{\xi} Y\right)=-\alpha([X,[\xi, Y]])=\alpha([Y,[X, \xi]])+\alpha([\xi,[Y, X]])=0 .
$$


Hence if $\mathrm{d} \alpha(X, Z)=0$ for any $Z \in \operatorname{ker}_{\xi}$, then $\mathrm{d} \alpha(X, W)=0$ for any $W \in \operatorname{ker} \alpha$ and consequently $X=0$. It follows that the restriction of $\alpha$ to ker $\operatorname{ad}_{\xi}$ is a contact form and $\xi$ is still the Reeb vector. Clearly $\xi$ belongs to the center of this subalgebra and the restrictions of $\Phi$ and $g$ induce a Sasakian structure on ker $\operatorname{ad}_{\xi}$.

(2) We can write

$$
X=a \xi+X^{\prime}, \quad Y=\left[\xi, Y^{\prime}\right]
$$

with $a \in \mathbb{R}, X^{\prime} \in \operatorname{ker} \operatorname{ad}_{\xi} \cap \operatorname{ker} \alpha, Y^{\prime} \in \mathfrak{g}$. Then, by using Jacobi identity,

$$
[X, Y]=a\left[\xi,\left[\xi, Y^{\prime}\right]\right]+\left[X^{\prime},\left[\xi, Y^{\prime}\right]\right]=a\left[\xi,\left[\xi, Y^{\prime}\right]\right]-\left[\xi,\left[Y^{\prime}, X^{\prime}\right]\right]
$$

which belongs to $\operatorname{Im} \operatorname{ad}_{\xi}$.

Remark 3.14. Note that if $\operatorname{ker} \alpha=\operatorname{Im~ad}_{\xi}$, then the commutator ideal $\mathfrak{g}^{\prime}=[\mathfrak{g}, \mathfrak{g}]$ coincides with $\mathfrak{g}$.

Assume now that the Sasakian Lie algebra $(\mathfrak{g}, \Phi, \alpha, \xi, g)$ with trivial center satisfies in addition the condition $\mathfrak{g}^{\prime} \neq \mathfrak{g}$.

With respect to the decomposition $\mathfrak{g}=\operatorname{ker} \operatorname{ad}_{\xi} \oplus \operatorname{Imad}_{\xi}$, we can write

$$
\left(\operatorname{ad}_{\xi}\right)_{\mid \operatorname{ker} \alpha}=\left(\begin{array}{cc}
0 & 0 \\
0 & U
\end{array}\right), \quad \Phi_{\mid \operatorname{ker} \alpha}=\left(\begin{array}{cc}
A & C \\
B & D
\end{array}\right),
$$

where $U: \operatorname{Imad}_{\xi} \rightarrow \operatorname{Imad}_{\xi}$ is a non-singular operator. Therefore from the equality $\Phi \operatorname{ad}_{\xi}=$ $\operatorname{ad}_{\xi} \Phi$ one gets that

and since $\left(\Phi_{\mid \operatorname{ker} \alpha}\right)^{2}=-\mathrm{I}$,

$$
B=C=0, \quad D U=U D,
$$

$$
A^{2}=D^{2}=-\mathrm{I} \text {. }
$$

In particular, if $\mathfrak{g}$ is solvable, then the Reeb vector $\xi$ cannot belong to the commutator $\mathfrak{g}^{\prime}$.

3.3. 3-dimensional Sasakian Lie algebras. Simply connected homogeneous 3dimensional contact metric manifolds were classified by Perrone in [27], showing that the homogeneous space has to be a Lie group with a left-invariant contact metric structure. Among these Lie groups we can find the ones that admit a Sasakian structure.

For the sake of completeness we perform the classification of 3-dimensional Sasakian Lie groups even if it is already known also by [18, 10]. Indeed, Geiges in [18] has determined the diffeomorphism types of compact manifolds of dimension 3 that admit a Sasakian structure.

Theorem 3.15. Any 3-dimensional Sasakian Lie algebra is isomorphic to one of the following: $\mathfrak{s u}(2), \mathfrak{s l}(2, \mathbb{R}), \mathfrak{a f f}(\mathbb{R}) \times \mathbb{R}, \mathfrak{h}_{3}$.

Proof. It is well known that, if the commutator $\mathfrak{g}^{\prime}$ of a 3 -dimensional Lie algebra $\mathfrak{g}$ coincides with $\mathfrak{g}$, then $\mathfrak{g}$ is semisimple and it is isomorphic to either $\mathfrak{s u}(2)$ or $\mathfrak{s l}(2, \mathbb{R})$. Both Lie algebras admit a Sasakian structure (see for instance [18, 10, 27]).

If $\mathfrak{g}^{\prime} \neq \mathfrak{g}$ then $\mathfrak{g}$ is solvable and, by Corollary 3.12 , we have the orthogonal decomposition

$$
\mathfrak{g}=\operatorname{ker} \operatorname{ad}_{\xi} \oplus \operatorname{Im} \operatorname{ad}_{\xi},
$$

where $\xi$ is the Reeb vector. Since $\operatorname{ker} \operatorname{ad}_{\xi} \cap \operatorname{ker} \alpha$ is $\Phi$-invariant, the only possibility is that $\operatorname{dim}\left(\operatorname{ker} \operatorname{ad}_{\xi} \cap \operatorname{ker} \alpha\right)=2$ and thus $\xi$ belongs to the center $\mathfrak{z}(\mathfrak{g})$. The Kähler quotient 
$\mathfrak{g} / \mathfrak{z}(\mathfrak{g})$ is then isomorphic to $\mathbb{R}^{2}$ or $\mathfrak{a} \mathfrak{f} \mathfrak{f}(\mathbb{R})$. It is easy to show that in the former case $\mathfrak{g}$ is isomorphic to $\mathfrak{h}_{3}$. In the latter case there exists a basis $\left\{e^{1}, e^{2}, e^{3}\right\}$ of $\mathfrak{g}^{*}$ such that

$$
\mathrm{d} e^{1}=0, \quad \mathrm{~d} e^{2}=e^{12}, \quad \mathrm{~d} e^{3}=2 e^{12},
$$

with respect to which the Sasakian structure is

$$
\xi=e_{3}, \quad \alpha=e^{3}, \quad \Phi\left(e_{1}\right)=e_{2}, \quad \omega=e^{12} .
$$

Considering the new basis

$$
E^{j}=e^{j}, j=1,2, \quad E^{3}=e^{3}-2 e^{2},
$$

we have the isomorphism of $\mathfrak{g}$ with $\mathfrak{a f f}(\mathbb{R}) \times \mathbb{R}$.

\section{5-Dimensional Sasakian Lie algebras}

In order to classify 5-dimensional Sasakian Lie algebras $\mathfrak{g}$ up to isomorphism we will consider separately the case of Lie algebras with trivial or non-trivial center.

4.1. 5-dimensional Sasakian Lie algebras with non-trivial center. Proposition 3.8 together with the classification in [25] of 4-dimensional Kähler Lie algebras allow us to classify 5-dimensional Sasakian Lie algebras with non-trivial center.

Theorem 4.1. Any 5-dimensional Sasakian Lie algebra $\mathfrak{g}$ with non-trivial center is isomorphic to one of the following Lie algebras

$$
\begin{aligned}
\mathfrak{g}_{1} & =\left(0,0,0,0, e^{12}+e^{34}\right) \simeq \mathfrak{h}_{5} ; \\
\mathfrak{g}_{2} & =\left(0,-e^{12}, 0,0, e^{12}+e^{34}\right) \simeq \mathfrak{a f f}(\mathbb{R}) \times \mathfrak{h}_{3} ; \\
\mathfrak{g}_{3} & =\left(0,-e^{13}, e^{12}, 0, e^{14}+e^{23}\right) \simeq \mathbb{R} \ltimes\left(\mathfrak{h}_{3} \times \mathbb{R}\right) ; \\
\mathfrak{g}_{4} & =\left(0,-e^{12}, 0,-e^{34}, e^{12}+e^{34}\right) \simeq \mathfrak{a f f}(\mathbb{R}) \times \mathfrak{a f f}(\mathbb{R}) \times \mathbb{R} ; \\
\mathfrak{g}_{5} & =\left(\frac{1}{2} e^{14}, \frac{1}{2} e^{24},-e^{12}+e^{34}, 0, e^{12}-e^{34}\right) \simeq \mathbb{R} \times\left(\mathbb{R} \ltimes \mathfrak{h}_{3}\right) ; \\
\mathfrak{g}_{6} & =\left(2 e^{14},-e^{24},-e^{12}+e^{34}, 0, e^{23}\right) \simeq \mathbb{R} \ltimes \mathfrak{n}_{4} ; \\
\mathfrak{g}_{7}^{\delta} & =\left(\frac{\delta}{2} e^{14}+e^{24},-e^{14}+\frac{\delta}{2} e^{24},-e^{12}+\delta e^{34}, 0, e^{12}-\delta e^{34}\right) \simeq \mathbb{R} \times\left(\mathbb{R} \ltimes \mathfrak{h}_{3}\right), \delta>0 ; \\
\mathfrak{g}_{8}^{\delta} & =\left(e^{14}, \delta e^{34},-\delta e^{24}, 0, e^{14}+e^{23}\right) \simeq \mathbb{R} \ltimes_{\delta}\left(\mathfrak{h}_{3} \times \mathbb{R}\right), \delta>0 .
\end{aligned}
$$

Proof. By Corollary [3.7, if $(\mathfrak{g}, \Phi, \alpha, \xi, g)$ is a 5-dimensional Sasakian Lie algebra, then the quotient $\mathfrak{g} / \mathfrak{z}(\mathfrak{g})$ is a 4-dimensional Kähler Lie algebra with the Kähler structure induced by the Sasakian one. We may choose a basis $\left\{e_{1}, \ldots, e_{5}\right\}$ of $\mathfrak{g}$ such that

$$
\xi=e_{5}, \quad \alpha=e^{5}, \quad \mathfrak{g} / \mathfrak{z}(\mathfrak{g})=\operatorname{Span}\left\{e_{1}, e_{2}, e_{3}, e_{4}\right\}
$$

and $\mathrm{d} e^{5}=2 \Omega$, where $\Omega$ is the Kähler form on the quotient. 
4-dimensional Kähler Lie algebras and their possible Kähler forms have been classified in [25]. By using this classification we obtain that $\mathfrak{g}$ is isomorphic to one of the following Lie algebras

$$
\begin{aligned}
\mathfrak{k}_{1} & =\left(0,0,0,0, \lambda e^{12}+\mu e^{34}\right), \quad \lambda, \mu<0 ; \\
\mathfrak{k}_{2} & =\left(0,-e^{12}, 0,0, \lambda e^{12}+\mu e^{34}\right), \quad \lambda, \mu<0 ; \\
\mathfrak{k}_{3} & =\left(0,-e^{13}, e^{12}, 0, \lambda e^{14}+\mu e^{23}\right), \quad \lambda, \mu<0 ; \\
\mathfrak{k}_{4} & =\left(0,-e^{12}, 0,-e^{34}, \lambda e^{12}+\mu e^{34}\right), \quad \lambda, \mu<0 ; \\
\mathfrak{k}_{5} & =\left(\frac{1}{2} e^{14}, \frac{1}{2} e^{24},-e^{12}+e^{34}, 0, \lambda\left(e^{12}-e^{34}\right)\right), \quad \lambda<0 ; \\
\mathfrak{k}_{6} & =\left(2 e^{14},-e^{24},-e^{12}+e^{34}, 0, \lambda e^{14}+\mu e^{23}\right), \quad \lambda, \mu<0 ; \\
\mathfrak{k}_{7}^{-} & =\left(\frac{\delta}{2} e^{14}+e^{24},-e^{14}+\frac{\delta}{2} e^{24},-e^{12}+\delta e^{34}, 0, \lambda\left(e^{12}-\delta e^{34}\right)\right), \quad \delta>0, \lambda<0 ; \\
\mathfrak{k}_{7}^{+} & =\left(\frac{\delta}{2} e^{14}+e^{24},-e^{14}+\frac{\delta}{2} e^{24},-e^{12}+\delta e^{34}, 0, \lambda\left(e^{12}-\delta e^{34}\right)\right), \quad \delta>0 ; \\
\mathfrak{k}_{8}^{-} & =\left(e^{14}, \delta e^{34},-\delta e^{24}, 0, \lambda e^{14}+\mu e^{23}\right), \quad \delta, \lambda>0, \mu<0 ; \\
\mathfrak{k}_{8}^{+} & =\left(e^{14}, \delta e^{34},-\delta e^{24}, 0, \lambda e^{14}+\mu e^{23}\right), \quad \delta, \lambda, \mu>0 ;
\end{aligned}
$$

equipped with the Sasakian structure $\left(\Phi, \alpha=e^{5}, \xi=e_{5}, g\right)$, where $\Phi$ is given respectively by

$$
\begin{array}{ll}
\Phi_{1}\left(e_{1}\right)=e_{2}, & \Phi_{1}\left(e_{3}\right)=e_{4} \\
\Phi_{2}\left(e_{1}\right)=e_{2}, & \Phi_{2}\left(e_{3}\right)=e_{4} \\
\Phi_{3}\left(e_{1}\right)=e_{4}, & \Phi_{3}\left(e_{2}\right)=e_{3} \\
\Phi_{4}\left(e_{1}\right)=e_{2}, & \Phi_{4}\left(e_{3}\right)=e_{4} \\
\Phi_{5}\left(e_{1}\right)=e_{2}, & \Phi_{5}\left(e_{4}\right)=e_{3} \\
\Phi_{6}\left(e_{2}\right)=e_{3}, & \Phi_{6}\left(e_{4}\right)=-2 e_{1} \\
\Phi_{7}^{-}\left(e_{1}\right)=e_{2}, & \Phi_{7}^{-}\left(e_{4}\right)=e_{3} \\
\Phi_{7}^{+}\left(e_{1}\right)=-e_{2}, & \Phi_{7}^{+}\left(e_{4}\right)=-e_{3} \\
\Phi_{8}^{-}\left(e_{4}\right)=e_{1}, & \Phi_{8}^{-}\left(e_{2}\right)=e_{3} \\
\Phi_{8}^{+}\left(e_{4}\right)=e_{1}, & \Phi_{8}^{+}\left(e_{3}\right)=e_{2}
\end{array}
$$

and $2 g(\cdot, \cdot)=\mathrm{d} e^{5}(\Phi \cdot, \cdot)$.

By a direct computation one may check the following isomorphisms

$$
\mathfrak{k}_{i} \cong \mathfrak{g}_{i}, i=1, \ldots, 5, \quad \mathfrak{k}_{6}^{ \pm} \cong \mathfrak{g}_{6}^{\tau}(\text { with } \tau=\lambda / \mu), \quad \mathfrak{k}_{j}^{ \pm} \cong \mathfrak{g}_{j}^{\delta}, j=7,8 .
$$

For the family

$$
\mathfrak{g}_{6}^{\tau}=\left(2 e^{14},-e^{24},-e^{12}+e^{34}, 0, \tau e^{14}+e^{23}\right), \tau>0
$$

we can show that every element in the family is isomorphic to $\mathfrak{g}_{6}$ by considering the new basis

$$
E_{1}=2 e_{1}+\tau e_{5}, \quad E_{j}=e_{j}, j=2, \ldots, 5 .
$$

By Proposition 3.8, the Lie algebras $\mathfrak{g}_{i}$ and $\mathfrak{g}_{j}^{\delta}$ are not isomorphic for any $i$ and $j$. Moreover, $\mathfrak{g}_{i}$ (respectively $\mathfrak{g}_{i}^{\delta}$ ) is not isomorphic to $\mathfrak{g}_{k}$ (respectively $\mathfrak{g}_{k}^{\delta}$ ) for any $i \neq k$. 
Applying again Proposition 3.8, it follows that the Lie algebras in the family $\mathfrak{g}_{7}^{\delta}$ are not isomorphic one each other for different values of $\delta$. The same holds for the family $\mathfrak{g}_{8}^{\delta}$.

As a consequence we have the following

Corollary 4.2. A unimodular Sasakian Lie algebra with non-trivial center is isomorphic either to the nilpotent Heisenberg Lie algebra $\mathfrak{h}_{5}$ or the solvable Lie algebra $\mathfrak{g}_{3}$. The simply connected Lie group $G_{3}$ with Lie algebra $\mathfrak{g}_{3}$ admits a co-compact discrete subgroup $\Gamma$.

Proof. By a direct computation one may check that the only unimodular Sasakian Lie algebras with non-trivial center are in fact $\mathfrak{g}_{1} \cong \mathfrak{h}_{5}$ and $\mathfrak{g}_{3} \cong \mathbb{R} \ltimes\left(\mathfrak{h}_{3} \times \mathbb{R}\right)$. The solvable simply connected Lie group $G_{3}$ is isomorphic to $\mathbb{R}^{5}$ with the following product

$$
\left(\begin{array}{l}
x_{1} \\
x_{2} \\
x_{3} \\
x_{4} \\
x_{5}
\end{array}\right) \cdot\left(\begin{array}{l}
y_{1} \\
y_{2} \\
y_{3} \\
y_{4} \\
y_{5}
\end{array}\right)=\left(\begin{array}{c}
x_{1}+y_{1} \\
\cos \left(x_{1}\right) y_{2}+\sin \left(x_{1}\right) y_{3}+x_{2} \\
-\sin \left(x_{1}\right) y_{2}+\cos \left(x_{1}\right) y_{3}+x_{3} \\
Q \\
x_{5}+y_{5}
\end{array}\right)
$$

where

$$
Q=y_{4}+x_{1} y_{5}-\sin ^{2}\left(x_{1}\right) y_{2} y_{3}-\frac{1}{4} \sin \left(2 x_{1}\right)\left(y_{2}^{2}-y_{3}^{2}\right)+x_{2}\left(-\sin \left(x_{1}\right) y_{2}+\cos \left(x_{1}\right) y_{3}\right)+x_{4} \text {. }
$$

The discrete subgroup

$$
\Gamma=\left\{\left(2 \pi m_{1}, m_{2}, m_{3}, m_{4}, \frac{1}{2 \pi} m_{5}\right) \mid m_{i} \in \mathbb{Z}\right\}
$$

acts freely and properly discontinuously on $G_{3}$. Moreover, the quotient manifold $\Gamma \backslash G_{3}$ is compact.

The solvmanifold $\Gamma \backslash G_{3}$ is by construction the total space of an $S^{1}$-bundle over a 4dimensional non completely solvable Kähler solvmanifold. This class of Kähler solvmanifolds was found by Hasegawa in [19] (see also [20]); all of these solvmanifolds are hyperelliptic surfaces. We recall that a solvmanifold is called completely solvable if the adjoint representation of the corresponding solvable Lie group has only real eigenvalues.

4.2. 5-dimensional Sasakian Lie algebras with trivial center. Let $(\mathfrak{g}, \Phi, \alpha, \xi, g)$ be a 5-dimensional Sasakian Lie algebra with trivial center and $\mathfrak{g}^{\prime}=\mathfrak{g}$. By [14, Section 5] the only contact Lie algebra with the above property is the semidirect product $\mathfrak{s l}(2, \mathbb{R}) \ltimes \mathbb{R}^{2}$, where $\mathfrak{s l}(2, \mathbb{R})$ acts on $\mathbb{R}^{2}$ by matrix multiplication. We can prove the following

Proposition 4.3. The Lie algebra $\mathfrak{s l}(2, \mathbb{R}) \ltimes \mathbb{R}^{2}$ does not admit any Sasakian structure.

Proof. Let $\left\{e_{1}, e_{2}, e_{3}\right\}$ be the standard basis of $\mathfrak{s l}(2, \mathbb{R})$ with Lie brackets

$$
\left[e_{1}, e_{2}\right]=2 e_{2}, \quad\left[e_{1}, e_{3}\right]=-2 e_{3}, \quad\left[e_{2}, e_{3}\right]=e_{1}
$$


and let $\left\{e_{4}, e_{5}\right\}$ be the canonical basis of $\mathbb{R}^{2}$. Then $\mathfrak{s l}(2, \mathbb{R}) \ltimes \mathbb{R}^{2}$ has structure equations

$$
\begin{aligned}
& \mathrm{d} e^{1}=-e^{23} \\
& \mathrm{~d} e^{2}=-2 e^{12} \\
& \mathrm{~d} e^{3}=2 e^{13} \\
& \mathrm{~d} e^{4}=-e^{14}-e^{25} \\
& \mathrm{~d} e^{5}=e^{15}-e^{34}
\end{aligned}
$$

A 1-form $\alpha=\sum_{i=1}^{5} a_{i} e^{i}$ is contact if and only if the real numbers $a_{i}$ satisfy the condition

$$
\Delta:=a_{3} a_{4}^{2}-a_{2} a_{5}^{2}-a_{1} a_{4} a_{5} \neq 0 .
$$

In this case, the corresponding Reeb vector is given by

$$
\xi=-\frac{1}{3 \Delta}\left(a_{4} a_{5} e_{1}+a_{5}^{2} e_{2}-a_{4}^{2} e_{3}+\left(a_{1} a_{5}-2 a_{3} a_{4}\right) e_{4}+\left(a_{1} a_{4}+2 a_{2} a_{5}\right) e_{5}\right) .
$$

One can check that $X \in \mathfrak{s l}(2, \mathbb{R}) \ltimes \mathbb{R}^{2}$ belongs to ker $\operatorname{ad}_{\xi} \cap \operatorname{ker} \alpha$ if and only

$$
X=t\left(-a_{5} e_{4}+a_{4} e_{5}\right), \quad t \in \mathbb{R} .
$$

If there exists a Sasakian structure with contact form $\alpha$, then $\operatorname{ker} \operatorname{ad}_{\xi} \cap \operatorname{ker} \alpha$ has to be $\Phi$-invariant (see Proposition 3.11) and this is only possible if $a_{4}=a_{5}=0$ in contrast with (7).

Now we can consider the case of 5-dimensional Sasakian Lie algebras with trivial center and such that $\mathfrak{g}^{\prime} \neq \mathfrak{g}$. In this case

$$
\operatorname{dim} \operatorname{ker}\left(\operatorname{ad}_{\xi}\right)_{\mid \operatorname{ker} \alpha}=\operatorname{dim} \operatorname{Im}\left(\operatorname{ad}_{\xi}\right)=2 .
$$

It is easy to see that there exists an orthonormal basis $\left\{e_{1}, \ldots, e_{4}\right\}$ of ker $\alpha$ with respect to which $\Phi_{\mid \text {ker } \alpha}$ can be written as

$$
\Phi_{\mid \text {ker } \alpha}=\left(\begin{array}{cccc}
0 & 1 & 0 & 0 \\
-1 & 0 & 0 & 0 \\
0 & 0 & 0 & 1 \\
0 & 0 & -1 & 0
\end{array}\right)
$$

and $\operatorname{kerad}_{\xi}=\operatorname{Span}\left\{\xi, e_{1}, e_{2}\right\}, \operatorname{Imad} \operatorname{ad}_{\xi}=\operatorname{Span}\left\{e_{3}, e_{4}\right\}$. Moreover in this basis

$$
\left(\operatorname{ad}_{\xi}\right)_{\mid \operatorname{ker} \alpha}=\left(\begin{array}{cccc}
0 & 0 & 0 & 0 \\
0 & 0 & 0 & 0 \\
0 & 0 & a & -b \\
0 & 0 & b & a
\end{array}\right)
$$

Note that in terms of $\left\{e_{1}, \ldots, e_{4}\right\}$ the 2 -form $\mathrm{d} \alpha$ takes the standard form $\mathrm{d} \alpha=2\left(e^{12}+e^{34}\right)$. Furthermore, taking into account that $\alpha\left(\left[e_{3}, e_{4}\right]\right)=-\mathrm{d} \alpha\left(e_{3}, e_{4}\right)=-2$, and recalling that 
$\theta: \mathfrak{g} \times \mathfrak{g} \rightarrow \operatorname{ker} \alpha$ denotes the projection of the bracket on $\mathfrak{g}$ onto ker $\alpha$, we have

$$
\begin{aligned}
0 & =\left[\xi,\left[e_{3}, e_{4}\right]\right]+\left[e_{4},\left[\xi, e_{3}\right]\right]-\left[e_{3},\left[\xi, e_{4}\right]\right] \\
& =\left[\xi, \theta\left(e_{3}, e_{4}\right)-2 \xi\right]+\left[e_{4}, a e_{3}+b e_{4}\right]-\left[e_{3},-b e_{3}+a e_{4}\right] \\
& =\left[\xi, \theta\left(e_{3}, e_{4}\right)\right]+a\left[e_{4}, e_{3}\right]-a\left[e_{3}, e_{4}\right] \\
& =\left[\xi, \theta\left(e_{3}, e_{4}\right)\right]-2 a\left[e_{3}, e_{4}\right] \\
& =\left[\xi, \theta\left(e_{3}, e_{4}\right)\right]-2 a \theta\left(e_{3}, e_{4}\right)+4 a \xi
\end{aligned}
$$

which implies

$$
a=0, \quad \theta\left(e_{3}, e_{4}\right) \in \operatorname{ker} \operatorname{ad}_{\xi} \cap \operatorname{ker} \alpha .
$$

From now on we set $e_{5}=\xi$ and denote by $\left\{e^{1}, \ldots, e^{5}\right\}$ the dual basis of $\left\{e_{1}, \ldots, e_{5}\right\}$. Since $b \neq 0$, by a suitable rescaling of $\alpha$ and $\xi$ we may assume $b= \pm 1$. We will examine separately the two cases: Case A: $b=1$ and Case B: $b=-1$.

We start by considering the case A. Taking into account (8) and Proposition [3.13, we can write

$$
\begin{aligned}
& \mathrm{d} e^{1}=a_{1} e^{12}+a_{6} e^{34}, \\
& \mathrm{~d} e^{2}=b_{1} e^{12}+b_{6} e^{34}, \\
& \mathrm{~d} e^{3}=-e^{45}+c_{2} e^{13}+c_{3} e^{14}+c_{4} e^{23}+c_{5} e^{24}, \\
& \mathrm{~d} e^{4}=e^{35}+f_{2} e^{13}+f_{3} e^{14}+f_{4} e^{23}+f_{5} e^{24}, \\
& \mathrm{~d} e^{5}=2\left(e^{12}+e^{34}\right) .
\end{aligned}
$$

From the condition $N_{\Phi}=-\mathrm{d} e^{5} \otimes e_{5}$ we get

$$
c_{5}=c_{2}-f_{3}-f_{4}, \quad f_{5}=f_{2}+c_{3}+c_{4} .
$$

Now we impose $\mathrm{d}^{2}=0$. From the vanishing of the coefficients of $e^{i j 5}, i, j=1, \ldots, 4$, in $\mathrm{d}^{2} e^{k}=0, k=1, \ldots, 5$, we get the following linear equations

$$
c_{2}-f_{3}=f_{2}+c_{3}=0 \text {. }
$$

Moreover,

$$
\mathrm{d}^{2} e^{5}=\left(a_{6}+2 c_{4}+f_{2}+c_{3}\right) e^{234}+\left(-b_{6}+c_{2}+f_{3}\right) e^{134} .
$$

and therefore in addition to (10) we have

$$
a_{6}=-2 c_{4}, \quad b_{6}=2 c_{2} .
$$

In this way the structure equations (9) of $\mathfrak{g}$ reduce to

$$
\begin{aligned}
& \mathrm{d} e^{1}=a_{1} e^{12}-2 c_{4} e^{34}, \\
& \mathrm{~d} e^{2}=b_{1} e^{12}+2 c_{2} e^{34}, \\
& \mathrm{~d} e^{3}=-e^{45}+c_{2} e^{13}+c_{3} e^{14}+c_{4} e^{23}-f_{4} e^{24}, \\
& \mathrm{~d} e^{4}=e^{35}-c_{3} e^{13}+c_{2} e^{14}+f_{4} e^{23}+c_{4} e^{24}, \\
& \mathrm{~d} e^{5}=2\left(e^{12}+e^{34}\right)
\end{aligned}
$$

with the structure constants satisfying the conditions

$$
\begin{aligned}
& c_{2}\left(a_{1}+2 c_{4}\right)=0, \quad c_{4}\left(a_{1}+2 c_{4}\right)=0, \quad c_{2}\left(-b_{1}+2 c_{2}\right)=0, \\
& c_{4}\left(-b_{1}+2 c_{2}\right)=0, \quad a_{1} c_{3}-b_{1} f_{4}+2=0, \quad c_{2} a_{1}+c_{4} b_{1}=0 .
\end{aligned}
$$


We get the following solutions for the above system

$$
\begin{aligned}
& \text { A1) } b_{1}=c_{2}=c_{4}=0, c_{3} \neq 0, a_{1}=-\frac{2}{c_{3}}, \\
& \text { A2) } c_{2}=c_{4}=0, b_{1} \neq 0, f_{4}=\frac{2+a_{1} c_{3}}{b_{1}}, \\
& \text { A3) } b_{1}=c_{2}=0, a_{1} \neq 0, c_{3}=-\frac{2}{a_{1}}, c_{4}=-\frac{1}{2} a_{1}, \\
& \text { A4) } b_{1} \neq 0, c_{2}=\frac{1}{2} b_{1}, c_{4}=-\frac{1}{2} a_{1}, f_{4}=\frac{2+a_{1} c_{3}}{b_{1}} .
\end{aligned}
$$

In the first two cases, we have that

$$
\mathrm{A} 1), \mathrm{A} 2) \simeq \mathfrak{a} \mathfrak{f} \mathfrak{f}(\mathbb{R}) \times \mathfrak{s l}(2, \mathbb{R})
$$

where respectively

$$
\text { A1) }\left\{\begin{array}{l}
\mathfrak{a f f}(\mathbb{R}) \simeq \operatorname{Span}\left\{f_{4} e_{1}-c_{3} e_{2}, e_{1}-c_{3} e_{5}\right\} \\
\mathfrak{s l}(2, \mathbb{R}) \simeq \operatorname{Span}\left\{e_{3}, e_{4}, e_{5}\right\}
\end{array}\right.
$$

and

$$
\text { A2) }\left\{\begin{array}{l}
\mathfrak{a f f}(\mathbb{R}) \simeq \operatorname{Span}\left\{a_{1} e_{1}+b_{1} e_{2}+2 e_{5}, e_{1}-c_{3} e_{5}\right\} \\
\mathfrak{s l}(2, \mathbb{R}) \simeq \operatorname{Span}\left\{e_{3}, e_{4}, e_{5}\right\}
\end{array}\right.
$$

In the other cases we see that

$$
\mathrm{A} 3), \mathrm{A} 4) \cong \mathbb{R}^{2} \ltimes \mathfrak{h}_{3}
$$

by using for A3) the new basis

$$
\left\{E_{1}=a_{1} e_{1}+2 e_{5}, E_{2}=\frac{1}{a_{1}} e_{2}, E_{j}=e_{j}, j=3,4,5\right\},
$$

with $\mathbb{R}^{2}=\operatorname{Span}\left\{E_{2}, E_{5}\right\}, \mathfrak{h}_{3}=\operatorname{Span}\left\{E_{1}, E_{3}, E_{4}\right\}$ and

$$
\operatorname{ad}_{E_{2}}=\left(\begin{array}{ccc}
1 & 0 & 0 \\
0 & \frac{1}{2} & \frac{f_{4}}{a_{1}} \\
0 & -\frac{f_{4}}{a_{1}} & \frac{1}{2}
\end{array}\right), \quad \operatorname{ad}_{E_{5}}=\left(\begin{array}{ccc}
0 & 0 & 0 \\
0 & 0 & -1 \\
0 & 1 & 0
\end{array}\right) .
$$

For A4) we may choose the new basis

$$
\left\{F_{1}=a_{1} e_{1}+b_{1} e_{2}+2 e_{5}, F_{2}=\frac{1}{b_{1}} e_{1}, F_{j}=e_{j}, j=3,4,5\right\},
$$

with $\mathbb{R}^{2}=\operatorname{Span}\left\{F_{2}, F_{5}\right\}, \mathfrak{h}_{3}=\operatorname{Span}\left\{F_{1}, F_{3}, F_{4}\right\}$ and

$$
\operatorname{ad}_{F_{2}}=\left(\begin{array}{ccc}
-1 & 0 & 0 \\
0 & -\frac{1}{2} & -\frac{c_{3}}{b_{1}} \\
0 & \frac{c_{3}}{b_{1}} & -\frac{1}{2}
\end{array}\right), \quad \operatorname{ad}_{F_{5}}=\left(\begin{array}{ccc}
0 & 0 & 0 \\
0 & 0 & -1 \\
0 & 1 & 0
\end{array}\right) .
$$


Now we study the case B. Taking into account (8) and Proposition 3.13, we can write

$$
\begin{aligned}
& \mathrm{d} e^{1}=a_{1} e^{12}+a_{6} e^{34}, \\
& \mathrm{~d} e^{2}=b_{1} e^{12}+b_{6} e^{34}, \\
& \mathrm{~d} e^{3}=e^{45}+c_{2} e^{13}+c_{3} e^{14}+c_{4} e^{23}+c_{5} e^{24}, \\
& \mathrm{~d} e^{4}=-e^{35}+f_{2} e^{13}+f_{3} e^{14}+f_{4} e^{23}+f_{5} e^{24}, \\
& \mathrm{~d} e^{5}=2\left(e^{12}+e^{34}\right) .
\end{aligned}
$$

Condition $N_{\Phi}=-\mathrm{d} e^{5} \otimes e_{5}$ implies the following linear equations

$$
c_{5}=c_{2}-f_{3}-f_{4}, f_{5}=f_{2}+c_{3}+c_{4},
$$

while $\mathrm{d}^{2}=0$ gives

$$
c_{2}=f_{3}, f_{2}=-c_{3}, a_{6}=-2 c_{4}, f_{3}=\frac{1}{2} b_{6} .
$$

Hence the structure equations (11) of $\mathfrak{g}$ reduces to

$$
\begin{aligned}
\mathrm{d} e^{1} & =a_{1} e^{12}-2 c_{4} e^{34}, \\
\mathrm{~d} e^{2} & =b_{1} e^{12}+b_{6} e^{34}, \\
\mathrm{~d} e^{3} & =e^{45}+\frac{1}{2} b_{6} e^{13}+c_{3} e^{14}+c_{4} e^{23}-f_{4} e^{24}, \\
\mathrm{~d} e^{4} & =-e^{35}-c_{3} e^{13}+\frac{1}{2} b_{6} e^{14}+f_{4} e^{23}+c_{4} e^{24}, \\
\mathrm{~d} e^{5} & =2\left(e^{12}+e^{34}\right),
\end{aligned}
$$

where the structure constants are related by the equations

$$
\begin{aligned}
& c_{4}\left(2 c_{4}+a_{1}\right)=0, b_{6}\left(a_{1}+2 c_{4}\right)=0, c_{4}\left(b_{1}-b_{6}\right)=0, b_{6}\left(b_{6}-b_{1}\right)=0 \\
& b_{1} c_{4}+\frac{1}{2} a_{1} b_{6}=0, c_{3} a_{1}-b_{1} f_{4}-2=0 .
\end{aligned}
$$

This imposes to consider the following four cases:

$$
\begin{aligned}
& \text { B1) } b_{1}=0, b_{6}=0, c_{4}=0, c_{3}=\frac{2}{a_{1}}, \\
& \text { B2) } b_{6}=0, c_{4}=0, f_{4}=\frac{c_{3} a_{1}-2}{b_{1}}, \\
& \text { B3) } b_{1}=0, b_{6}=0, c_{3}=\frac{2}{a_{1}}, c_{4}=-\frac{1}{2} a_{1}, \\
& \text { B4) } b_{6}=b_{1}, c_{4}=-\frac{1}{2} a_{1}, f_{4}=\frac{c_{3} a_{1}-2}{b_{1}} .
\end{aligned}
$$

In the first two cases we have

$$
\mathrm{B} 1), \mathrm{B} 2) \simeq \mathfrak{a f f}(\mathbb{R}) \times \mathfrak{s u}(2),
$$

where respectively

$$
\text { B1) }\left\{\begin{array}{l}
\mathfrak{a f f}(\mathbb{R}) \simeq \operatorname{Span}\left\{a_{1} e_{1}+e_{5}, f_{4} e_{1}+e_{5}\right\} \\
\mathfrak{s u}(2) \simeq \operatorname{Span}\left\{e_{3}, e_{4}, e_{5}\right\}
\end{array}\right.
$$


and

$$
\text { B2) }\left\{\begin{array}{l}
\mathfrak{a f f}(\mathbb{R}) \simeq \operatorname{Span}\left\{a_{1} e_{1}+b_{1} e_{2}+2 e_{5}, e_{1}+c_{3} e_{5}\right\} \\
\mathfrak{s u}(2) \simeq \operatorname{Span}\left\{e_{3}, e_{4}, e_{5}\right\}
\end{array}\right.
$$

Again in the cases B3) and B4) $\mathfrak{g}$ is solvable and

$$
\mathrm{B} 3), \mathrm{B} 4) \cong \mathbb{R}^{2} \ltimes \mathfrak{h}_{3}
$$

by using for B3) the new basis

$$
\left\{G_{1}=a_{1} e_{1}+2 e_{5}, G_{2}=\frac{1}{a_{1}} e_{2}, G_{j}=e_{j}, j=3,4,5\right\},
$$

with $\mathbb{R}^{2}=\operatorname{Span}\left\{G_{2}, G_{5}\right\}, \mathfrak{h}_{3}=\operatorname{Span}\left\{G_{1}, G_{3}, G_{4}\right\}$ and

$$
\operatorname{ad}_{G_{2}}=\left(\begin{array}{ccc}
1 & 0 & 0 \\
0 & \frac{1}{2} & \frac{f_{4}}{a_{1}} \\
0 & -\frac{f_{4}}{a_{1}} & \frac{1}{2}
\end{array}\right), \quad \operatorname{ad}_{G_{5}}=\left(\begin{array}{ccc}
0 & 0 & 0 \\
0 & 0 & 1 \\
0 & -1 & 0
\end{array}\right) .
$$

For B4) we may choose the new basis

$$
\left\{H_{1}=a_{1} e_{1}+b_{1} e_{2}+2 e_{5}, H_{2}=\frac{1}{b_{1}} e_{1}, H_{j}=e_{j}, j=3,4,5\right\},
$$

with $\mathbb{R}^{2}=\operatorname{Span}\left\{H_{2}, H_{5}\right\}, \mathfrak{h}_{3}=\operatorname{Span}\left\{H_{1}, H_{3}, H_{4}\right\}$ and

$$
\operatorname{ad}_{H_{2}}=\left(\begin{array}{ccc}
-1 & 0 & 0 \\
0 & -\frac{1}{2} & -\frac{c_{3}}{b_{1}} \\
0 & \frac{c_{3}}{b_{1}} & -\frac{1}{2}
\end{array}\right), \quad \operatorname{ad}_{H_{5}}=\left(\begin{array}{ccc}
0 & 0 & 0 \\
0 & 0 & 1 \\
0 & -1 & 0
\end{array}\right) .
$$

In the cases A3), A4), B3), B4), the corresponding Lie algebras are all isomorphic to a semidirect product $\mathfrak{g}_{t}=\mathbb{R}^{2} \ltimes_{\psi_{t}} \mathfrak{h}_{3}$, where $\mathbb{R}^{2}=\operatorname{Span}\{X, Y\}, \mathfrak{h}_{3}=\operatorname{Span}\left\{v_{1}, v_{2}, v_{3}\right\}$ and

$$
\left[v_{2}, v_{3}\right]=-v_{1}, \quad \psi_{t}(X)=\left(\begin{array}{ccc}
1 & 0 & 0 \\
0 & \frac{1}{2} & t \\
0 & -t & \frac{1}{2}
\end{array}\right), \quad \psi_{t}(Y)=\left(\begin{array}{ccc}
0 & 0 & 0 \\
0 & 0 & -1 \\
0 & 1 & 0
\end{array}\right)
$$

By changing $X$ to $X+t Y$ we obtain an isomorphism between $\mathfrak{g}_{t}$ and $\mathfrak{g}_{0}$ with structure equations (11).

To summarize we can state the following

Theorem 4.4. If a 5-dimensional Sasakian Lie algebra $\mathfrak{g}$ has trivial center, then it is isomorphic to one of the following Lie algebras: the direct products $\mathfrak{s l}(2, \mathbb{R}) \times \mathfrak{a f f}(\mathbb{R})$, $\mathfrak{s u}(2) \times \mathfrak{a f f}(\mathbb{R})$, or the non-unimodular solvable Lie algebra $\mathfrak{g}_{0}$.

Remark 4.5. Note that $\mathfrak{g}_{0}$ corresponds to the Lie algebra numbered 22 in the classification of 5 -dimensional solvable contact Lie algebras provided by Diatta in [14]. 


\section{5-Dimensional SASAKIAN $\alpha$-Einstein LiE ALGEBRAS}

In this section we study Sasakian $\alpha$-Einstein Lie algebras. A Sasakian Lie algebra $(\mathfrak{g}, \Phi, \alpha, \xi, g)$ is called $\alpha$-Einstein if the Ricci tensor $\operatorname{Ric}_{g}$ of the metric $g$ satisfies $\operatorname{Ric}_{g}=$ $\lambda g+\nu \alpha \otimes \alpha$ for some $\lambda, \nu \in \mathbb{R}$.

It is known that the canonical Sasakian structure on $\mathfrak{h}_{5}$ is $\alpha$-Einstein. Furthermore, in view of [1, Proposition 4.2] the Lie algebra $\mathfrak{g}_{0}$ from Theorem 4.4 is the only solvable (non nilpotent) 5-dimensional Lie algebra admitting a Sasakian $\alpha$-Einstein structure. Thus, in order to determine all the 5-dimensional Lie algebras admitting such a structure, we only have to consider the non-solvable ones, which are $\mathfrak{s l}(2, \mathbb{R}) \times \mathfrak{a f f}(\mathbb{R})$ and $\mathfrak{s u}(2) \times \mathfrak{a f f}(\mathbb{R})$ according to Theorems 4.1 and 4.4 .

Proposition 5.1. The Lie algebra $\mathfrak{s l}(2, \mathbb{R}) \times \mathfrak{a f f}(\mathbb{R})$ admits Sasakian $\alpha$-Einstein structures, while there are none Sasakian $\alpha$-Einstein structures on $\mathfrak{s u}(2) \times \mathfrak{a f f}(\mathbb{R})$.

Proof. All Sasakian structures on $\mathfrak{s l}(2, \mathbb{R}) \times \mathfrak{a f f}(\mathbb{R})$ are described in the cases A1), A2) given in 4.2 . Performing standard computations we obtain that in the case A1) the Ricci tensor is given by the following matrix

$$
\operatorname{Ric}_{g}=\left(\begin{array}{ccccc}
-\frac{2 c_{3}^{2}+2}{c_{3}^{2}} & 0 & 0 & 0 & 0 \\
0 & -\frac{2 c_{3}^{2}+2}{c_{3}^{2}} & 0 & 0 & 0 \\
0 & 0 & -4 & 0 & 0 \\
0 & 0 & 0 & -4 & 0 \\
0 & 0 & 0 & 0 & 4
\end{array}\right)
$$

Therefore for $c_{3}= \pm 1$ we see that the Sasakian structure is $\alpha$-Einstein. In the case A2) we have

$$
\operatorname{Ric}_{g}=\left(\begin{array}{ccccc}
-\left(2+a_{1}^{2}+b_{1}^{2}\right) & 0 & 0 & 0 & 0 \\
0 & -\left(2+a_{1}^{2}+b_{1}^{2}\right) & 0 & 0 & 0 \\
0 & 0 & -4 & 0 & 0 \\
0 & 0 & 0 & -4 & 0 \\
0 & 0 & 0 & 0 & 4
\end{array}\right) .
$$

Therefore for $a_{1}^{2}+b_{1}^{2}=2$ we obtain that the Sasakian structure is $\alpha$-Einstein.

We consider now the Lie algebra $\mathfrak{s u}(2) \times \mathfrak{a f f}(\mathbb{R})$. For this algebra the Sasakian structures are described by the cases B1), B2) studied in 4.2 . In the case B1) the Ricci tensor is given by

$$
\operatorname{Ric}_{g}=\left(\begin{array}{ccccc}
-\left(2+a_{1}^{2}\right) & 0 & 0 & 0 & 0 \\
0 & -\left(2+a_{1}^{2}\right) & 0 & 0 & 0 \\
0 & 0 & 0 & 0 & 0 \\
0 & 0 & 0 & 0 & 0 \\
0 & 0 & 0 & 0 & 4
\end{array}\right)
$$


whereas in the case B2) it is given by

$$
\operatorname{Ric}_{g}=\left(\begin{array}{ccccc}
-\left(2+a_{1}^{2}+b_{1}^{2}\right) & 0 & 0 & 0 & 0 \\
0 & -\left(2+a_{1}^{2}+b_{1}^{2}\right) & 0 & 0 & 0 \\
0 & 0 & 0 & 0 & 0 \\
0 & 0 & 0 & 0 & 0 \\
0 & 0 & 0 & 0 & 4
\end{array}\right) .
$$

As a consequence the Sasakian structures on this Lie algebra never satisfy the $\alpha$-Einstein condition.

To sum up, we can now state the following

Theorem 5.2. The only 5-dimensional Lie algebras admitting a Sasakian $\alpha$-Einstein structure are $\mathfrak{h}_{5}, \mathfrak{g}_{0}$ and $\mathfrak{s l}(2, \mathbb{R}) \times \mathfrak{a f f}(\mathbb{R})$.

\section{REFERENCES}

[1] de Andrés L. C., Fernández M., Fino A., Ugarte L., Contact 5-manifolds with SU(2)-structure, preprint arXiv:0706.0386, to appear in Q. J. Math.

[2] Blair D.E.: Riemannian Geometry of Contact and Symplectic Manifolds, Birkhäuser (2002).

[3] Boeckx, E., A full classification of contact metric $(\kappa, \mu)$-spaces, Illinois J. Math. 44 (2000), 212-219.

[4] Boothby W. M., Wang H. C.: On contact manifolds, Ann. of Math. (2) 68 (1958), 721-734.

[5] Boyer C. P., Galicki K.: 3-Sasakian manifolds, Surveys in differential geometry: essays on Einstein manifolds 123-184, Surv. Differ. Geom., VI, Int. Press, Boston, MA, 1999.

[6] Boyer C. P., Galicki, K.: On Sasakian-Einstein geometry, Internat. J. Math. 11 (2000), 873-909.

[7] Boyer C. P., Galicki K., Kollár J.: Einstein metrics on spheres, Ann. of Math. (2) 162 (2005), no. 1, $557-580$.

[8] Boyer C.P, Galicki K., Matzeu P.: On Eta-Einstein Sasakian Geometry, Commun. Math. Phys. 262 (2006), 177-208.

[9] Boyer C. P., Galicki K., Ornea L.: Constructions in Sasakian geometry, Math. Z. 257 (2007), no. 4, 907-924.

[10] Cho, J. T., Chun S. H., On the classification of contact Riemannian manifolds satisfying the condition (C), Glasg. Math. J. 45 (2003), 475-492.

[11] van Coevering C.: Toric Surfaces and Sasakian-Einstein 5-manifolds, preprint arXiv:math/0607721 $\mathrm{PhD}$ dissertation.

[12] Conti D.: Cohomogeneity one Einstein-Sasaki 5-manifolds, Comm. Math. Phys. 274 (2007), no. 3, 751-774.

[13] Conti D., Salamon S.: Generalized Killing spinors in dimension 5, Trans. Amer. Math. Soc. 359 (2007), 5319-5343.

[14] Diatta A.: Left-invariant contact structures on Lie groups, Diff. Geom. Appl. 26 (2008), no. 5, $544-552$.

[15] Diatta A.: Riemannian geometry on contact Lie groups, Geom. Dedicata 133 (2008), 83-94.

[16] Gauntlett J. P., Martelli D., Sparks J., Waldram D.: Sasaki-Einstein metrics on $S^{2} \times S^{3}$, Adv. Theor. Math. Phys. 8 (2004), no. 4, 711-734.

[17] Gauntlett J. P., Martelli D. Sparks J., Yau, S.-T.: Obstructions to the existence of Sasaki-Einstein metrics, Comm. Math. Phys. 273 (2007), 803-827.

[18] Geiges H., Normal contact structures on 3-manifolds, Tôhoku Math. J. (2) 49 (1997), 415-422.

[19] Hasegawa, K.: A class of compact Kählerian solvmanifolds and a general conjecture, Geom. Dedicata 78 (1999), 253-258.

[20] Hasegawa, K.: Complex and Kähler structures on compact solvmanifolds, Conference on Symplectic Topology, J. Symplectic Geom. 3 (2005), no. 4, 749-767. 
[21] Hasegawa K.: A note on compact solvmanifolds with Kähler structures, Osaka J. Math. 43 (2006), 131-135.

[22] Hano J.: On Kählerian homogeneous spaces of unimodular Lie groups, Amer. J. Math. 79 (1957), 885-900.

[23] Okumura M.: Some remarks on space with a certain contact structure, Tôhoku Math. J. (2) (1962) 14, 135-145.

[24] Ornea L., Piccinni P.: Induced Hopf bundles and Einstein metrics, in New developments in differential geometry, Budapest (1996), 295-306, Kluwer.

[25] Ovando G.: Invariant pseudo-Kähler metrics in dimension four, J. Lie Theory 16 (2006), 371-391.

[26] Perrone D., Vanhecke L.: Five-dimensional homogeneous contact manifolds and related problems, Tôhoku Math. J. 43 (1991), 243-248.

[27] Perrone D.: Homogeneous contact Riemannian three-manifolds, Illinois J. Math. 42 (1998), 243-256.

[28] Sasaki S.: On differentiable manifolds with certain structures which are closely related to almost contact structure, Tôhoku Math. J. 2 (1960), 459-476.

[29] Sasaki, S.: Almost Contact Manifolds, Part 1. Lecture Notes, Mathematical Institute, Tôhoku University, 1965.

[30] Sasaki S., Hatakeyama Y.: On differentiable manifolds with certain structures which are closely related to almost contact structure II, Tôhoku Math. J. (2) 13 (1961), 281-294.

[31] Tomassini A., Vezzoni L.: Contact Calabi-Yau manifolds and Special Legendrian submanifolds, Osaka J. Math. 45 (2008), 127-147.

[32] Ugarte L.: Hermitian structures on six-dimensional nilmanifolds, Transform. Groups 12 (2007), 175202.

[33] Vaisman I.: Locally conformal Kähler manifolds with parallel Lee form, Rend. Mat. (6) 12 (1979), 263-284.

Adrián Andrada: Ciem-Famaf, Universidad Nacional de Córdoba, 5000 Córdoba, ArGENTINA

E-mail address: andrada@mate.uncor.edu

Anna Fino, Luigi Vezzoni: Dipartimento di Matematica, Università di Torino, Via Carlo Alberto 10, 10123 Torino, ItAly

E-mail address: annamaria.fino@unito.it, luigi.vezzoni@unito.it 